\title{
Mixed Strong-Electroweak Corrections to the Drell-Yan Process
}

\author{
Roberto Bonciani ${ }^{1,{ }^{*}}$ Luca Buonocore $\odot,{ }^{2, \dagger}$ Massimiliano Grazzini $\odot,{ }^{2, \ddagger}$ Stefan Kallweit $\odot, \$$ \\ Narayan Rana $\odot^{4, \|}$ Francesco Tramontano $\odot^{5, \pi}$ and Alessandro Vicini $\odot^{4, * *}$ \\ ${ }^{1}$ Dipartimento di Fisica, Università di Roma "La Sapienza" and INFN, Sezione di Roma, I-00185 Roma, Italy \\ ${ }^{2}$ Physik Institut, Universität Zürich, CH-8057 Zürich, Switzerland \\ ${ }^{3}$ Dipartimento di Fisica, Università degli Studi di Milano-Bicocca and INFN, Sezione di Milano-Bicocca, I-20126 Milan, Italy \\ ${ }^{4}$ Dipartimento di Fisica "Aldo Pontremoli", University of Milano and INFN, Sezione di Milano, I-20133 Milano, Italy \\ ${ }^{5}$ Dipartimento di Fisica, Università di Napoli Federico II and INFN, Sezione di Napoli, I-80126 Napoli, Italy
}

(Received 29 June 2021; accepted 9 December 2021; published 7 January 2022)

\begin{abstract}
We report on the first complete computation of the mixed QCD-electroweak (EW) corrections to the neutral-current Drell-Yan process. Superseding previously applied approximations, our calculation provides the first result at this order that is valid in the entire range of dilepton invariant masses. The two-loop virtual contribution is computed by using semianalytical techniques, overcoming the technical problems in the evaluation of the relevant master integrals. The cancellation of soft and collinear singularities is achieved by a formulation of the $q_{T}$ subtraction formalism valid in the presence of charged massive particles in the final state. We present numerical results for the fiducial cross section and selected kinematical distributions. At large values of the lepton $p_{T}$ the mixed QCD-EW corrections are negative and increase in size, to about $-15 \%$ with respect to the next-to-leading-order QCD result at $p_{T}=500 \mathrm{GeV}$. Up to dilepton invariant masses of $1 \mathrm{TeV}$ the computed corrections amount to about $-1.5 \%$ with respect to the next-to-leading-order QCD result.
\end{abstract}

DOI: 10.1103/PhysRevLett.128.012002

Introduction.-When the Large Hadron Collider (LHC) at CERN started data taking in 2009, it was expected to give answers to questions like the origin of electroweak (EW) symmetry breaking or the existence of supersymmetry. After successful physics runs at 7, 8, and $13 \mathrm{TeV}$ and the discovery of the Higgs boson [1,2], there is still no clear evidence of physics beyond the standard model. Although a huge amount of data will be accumulated in the highluminosity phase and exciting discoveries are still well possible, it is by now clear that an alternative path to uncover possible new physics is the search for small deviations from the predictions of the standard model, and that precision is the key for this path.

The Drell-Yan (DY) process [3] is the perfect example of a precision benchmark process at the LHC. It corresponds to the inclusive production of a lepton pair through an off shell vector boson. It provides large production rates and clean experimental signatures, given the presence of at least one lepton with large transverse momentum in the final state. Historically, it offered the first application of parton model ideas beyond deep inelastic scattering and led to the

Published by the American Physical Society under the terms of the Creative Commons Attribution 4.0 International license. Further distribution of this work must maintain attribution to the author(s) and the published article's title, journal citation, and DOI. Funded by SCOAP ${ }^{3}$. discovery of the $W$ and $Z$ bosons [4-7]. At present, the DY process provides valuable information about parton distribution functions, allows for the precise determination of several standard model parameters [8-11], and severely constrains many new-physics scenarios.

The DY process was one of the first hadronic reactions for which radiative corrections in the strong and EW couplings $\alpha_{S}$ and $\alpha$ were computed. The classic calculations of the next-to-leading-order (NLO) [12] and next-tonext-to-leading-order (NNLO) $[13,14]$ corrections to the total cross section in quantum chromodynamics (QCD) were followed by (fully) differential NNLO computations including the leptonic decay of the vector boson [15-19]. The complete EW corrections for $W$ production have been computed in Refs. [20-24], and for $Z$ production in Refs. [25-29]. Very recently, the next-to-next-to-next-toleading-order $\left(\mathrm{N}^{3} \mathrm{LO}\right) \mathrm{QCD}$ radiative calculations of the inclusive production of a virtual photon [30,31] and of a $W$ boson [32] have been completed, and first estimates of fiducial cross sections for the neutral-current DY process at the same order have appeared [33].

Since the high-precision determination of EW parameters requires control over the kinematical distributions at very high accuracy, the attention of the theory community has recently turned to the mixed QCD-EW corrections. The knowledge of these corrections would indeed allow us to improve over the approximations offered by shower Monte Carlo programs (see, e.g., Refs. [34,35] and 
Sec. IV 1 of Ref. [36]), which include only partial subsets of factorizable mixed QCD-EW corrections, and to reduce the remaining theoretical uncertainties.

The mixed QCD-QED corrections to the inclusive production of an on shell $Z$ boson were obtained in Ref. [37] through an abelianization procedure from the NNLO QCD results $[13,14]$. This calculation was extended to the fully differential level for off shell $Z$ boson production and decay into a pair of neutrinos (i.e., without final-state radiation) in Ref. [38]. A similar calculation was carried out in Ref. [39] in an on shell approximation for the $Z$ boson, but including the factorized NLO QCD corrections to $Z$ production and the NLO QED corrections to the leptonic $Z$ decay. Complete $\mathcal{O}\left(\alpha_{S} \alpha\right)$ computations for the production of on shell $Z$ and $W$ bosons have been presented in Refs. [40-44]. Beyond the on shell approximation, the most relevant results have been obtained in the pole approximation [45]. This approximation is based on a systematic expansion of the cross section around the $W$ or $Z$ resonance, in order to split the radiative corrections into well-defined, gauge-invariant contributions. Such a method has been used in Refs. $[46,47]$ to evaluate what is expected to be the dominant part of the mixed QCD-EW corrections in the resonance region.

Given the relevance of mixed QCD-EW corrections for precision studies of DY production and for an accurate measurement of the $W$ mass [48,49], it is important to go beyond this approximation. New-physics effects, in particular, could manifest themselves in the tails of kinematical distributions, where the pole approximation is not expected to work. A first step in this direction has been carried out in Ref. [50], where complete results for the $\mathcal{O}\left(n_{F} \alpha_{S} \alpha\right)$ contributions to the DY cross section were presented. Very recently, some of us have presented a computation [51] of the mixed QCD-EW corrections to the charged-current process $p p \rightarrow \ell \nu_{\ell}+X$, where all contributions are evaluated exactly except for the finite part of the two-loop amplitude, which was evaluated in the pole approximation.

One of the bottlenecks for a complete $\mathcal{O}\left(\alpha_{S} \alpha\right)$ calculation is indeed the corresponding two-loop virtual amplitude. The evaluation of the $2 \rightarrow 2$ two-loop Feynman diagrams with internal masses is at the frontier of current computational techniques. Progress on the evaluation of the corresponding two-loop master integrals has been reported in Refs. [52-57]. Very recently, the computation of the twoloop helicity amplitudes for neutral-current massless lepton pair production was discussed in Ref. [58]. In this Letter we report on an independent calculation of the two-loop amplitude and on its combination with the remaining perturbative contributions, to obtain the first complete computation of the mixed QCD-EW corrections for the neutral-current DY process.

The calculation.-We consider the inclusive production of a charged-lepton pair in proton collisions,

$$
p p \rightarrow \ell^{+} \ell^{-}+X .
$$

The theoretical predictions for this process can be obtained as a convolution of the parton distribution functions for the incoming protons with the hard scattering partonic cross section. When QCD and EW radiative corrections are considered, the initial partons include (anti-)quarks, gluons, and photons.

The differential cross section for the process in Eq. (1) can be written as

$$
d \sigma=\sum_{m, n=0}^{\infty} d \sigma^{(m, n)}
$$

where $d \sigma^{(0,0)} \equiv d \sigma_{\mathrm{LO}}$ is the Born level contribution and $d \sigma^{(m, n)}$ the $\mathcal{O}\left(\alpha_{S}^{m} \alpha^{n}\right)$ correction. The mixed QCD-EW corrections correspond to the term $m=n=1$ in this expansion and include double-real, real-virtual, and purely virtual contributions. The corresponding tree-level and oneloop scattering amplitudes are computed with OPENLOOPS [59-61] and RECOLA [62-64], finding complete agreement. The two-loop amplitude is computed using the following method. The Feynman diagrams are generated with QGRAF [65]. Using an in-house FORM [66] program we compute the unpolarized interference with the tree-level diagrams. The computation is done consistently in $d$ space-time dimensions, using a naive anticommuting $\gamma_{5}$ [67] and the scheme proposed in Ref. [68]. The interference is therefore expressed in terms of dimensionally regularized scalar integrals that are reduced to the master integrals (MIs) using integration-by-parts [69,70] and Lorentzinvariance [71] identities, as implemented in the computer codes KIRA [72], LITERED [73], and REDUZE2 [74,75]. The resulting set of MIs is available in the literature [52-57]. In particular, for the MIs with massive-boson exchange we refer to the implementation given in Ref. [52], where they are expressed in terms of generalized polylogarithms and Chen-iterated integrals [76-79]. The numerical evaluation of the Chen-iterated structures is very complicated and not a viable solution for a practical implementation. Therefore, for the evaluation of the most complicated MIs (five twoloop box-type MIs with two massive lines) we employ the semianalytical method of Ref. [80], implemented in the MATHEMATICA -based program DIFFEXP [81]. We performed several numerical checks with GINAC [82], FIESTA [83], and py SECDEC [84], finding complete agreement within the respective numerical uncertainties [85].

The computation of the amplitude is organized by breaking it into different gauge-independent ultravioletrenormalized subsets of diagrams, defined by the different possible combinations of electric and weak charges. In the evaluation of the amplitude we keep the lepton mass wherever need to regularize the final-state collinear singularities [86]. The two-loop virtual amplitude is computed in the background-field gauge [88], which restores the validity 
of QED-like Ward identities in the full standard model. The evaluation of the relevant two-loop counterterms $[89,90]$ is given in terms of two-loop self-energy diagrams [50,91,92].

Even when all the amplitudes have been computed, the completion of the calculation remains a formidable task. Indeed, double-real, real-virtual and purely virtual contributions are separately infrared divergent, and a method to handle and cancel infrared singularities has to be worked out. In this Letter, we use a formulation of the $q_{T}$ subtraction formalism [93] derived from the NNLO QCD computation of heavy-quark production [94-96] through an appropriate abelianization procedure [37,97]. The same method has been recently applied to the chargedcurrent DY process [51]. According to the $q_{T}$ subtraction formalism [93] $d \sigma^{(m, n)}$ can be evaluated as

$$
d \sigma^{(m, n)}=\mathcal{H}^{(m, n)} \otimes d \sigma_{\mathrm{LO}}+\left[d \sigma_{R}^{(m, n)}-d \sigma_{\mathrm{CT}}^{(m, n)}\right] .
$$

The first term in Eq. (3) is obtained through a convolution (denoted by the symbol $\otimes$ ) of the perturbatively computable function $\mathcal{H}^{(m, n)}$ and the leading order (LO) cross section $d \sigma_{\mathrm{LO}}$, with respect to the longitudinal-momentum fractions of the colliding partons. The second term is the real contribution $d \sigma_{R}^{(m, n)}$, where the charged leptons are accompanied by additional QCD and/or QED radiation that produces a recoil with finite transverse momentum $q_{T}$. For $m+n=2$ such contribution can be evaluated by using the dipole subtraction formalism [98-105]. In the limit $q_{T} \rightarrow 0$ the real contribution $d \sigma_{R}^{(m, n)}$ is divergent, since the recoiling radiation becomes soft and/or collinear to the initial-state partons. Such divergence is canceled by the counterterm $d \sigma_{\mathrm{CT}}^{(m, n)}$, which eventually makes the cross section in Eq. (3) finite.

The required phase space generation and integration is carried out within the MATRIX framework [106]. The core of MATRIX is the Monte Carlo program MUNICH [107], which contains a fully automated implementation of the dipole subtraction method for massless and massive partons at NLO QCD [98-100] and NLO EW [101-105]. The $q_{T}$ subtraction method has been applied to several NNLO QCD computations for the production of colorless finalstate systems (see Ref. [106] and references therein), and to heavy-quark production [94-96], which correspond to the case $m=2, n=0$. The method has also been applied in Ref. [97] to study NLO EW corrections to the DY process, which represents the case $m=0, n=1$. Very recently, some of us have applied the method to the computation of mixed QCD-EW corrections to the charged-current DY process [51]. The structure of the coefficients $\mathcal{H}^{(1,1)}$ and $d \sigma_{\mathrm{CT}}^{(1,1)}$ can be derived from those controlling the NNLO QCD computation of heavy-quark production. The initialstate soft-collinear and purely collinear contributions were already presented in Ref. [38]. The fact that the final state is color neutral implies that final-state radiation is of pure QED origin. Therefore, the purely soft contributions have a simpler structure than the corresponding contributions entering the NNLO QCD computation of Refs. [94-96]. The final result for the infrared-subtracted two-loop contribution, which enters the coefficient $\mathcal{H}^{(1,1)}$, is evaluated numerically on a two-dimensional grid by using the tools HARMONICSUMS [108,109], GINAC [82], and POLYLOGTOOLS [110].

Results.-We consider the process $p p \rightarrow \mu^{+} \mu^{-}+X$ at the center-of-mass energy $\sqrt{s}=14 \mathrm{TeV}$. As for the EW couplings, we follow the setup of Ref. [47]. In particular, we use the $G_{\mu}$ scheme with $G_{F}=1.1663787 \times 10^{-5} \mathrm{GeV}^{-2}$ and set the on shell values of masses and widths to $m_{W, \mathrm{OS}}=80.385 \mathrm{GeV}, \quad m_{Z, \mathrm{OS}}=91.1876 \mathrm{GeV}, \quad \Gamma_{W, \mathrm{OS}}=$ $2.085 \mathrm{GeV}, \Gamma_{Z, \mathrm{OS}}=2.4952 \mathrm{GeV}$. Those values are translated to the corresponding pole values $m_{V}=m_{V, \mathrm{OS}} /$ $\sqrt{1+\Gamma_{V, \mathrm{OS}}^{2} / m_{V, \mathrm{OS}}^{2}}$ and $\Gamma_{V}=\Gamma_{V, \mathrm{OS}} / \sqrt{1+\Gamma_{V, \mathrm{OS}}^{2} / m_{V, \mathrm{OS}}^{2}}$, $V=W, Z$, from which $\alpha=\sqrt{2} G_{F} m_{W}^{2}\left(1-m_{W}^{2} / m_{Z}^{2}\right) / \pi$ is derived, and we use the complex-mass scheme [111] throughout [112]. The muon mass is fixed to $m_{\mu}=105.658369 \mathrm{MeV}$, and the pole masses of the top quark and the Higgs boson to $m_{t}=173.07 \mathrm{GeV}$ and $m_{H}=125.9 \mathrm{GeV}$, respectively. The Cabibbo-KobayashiMaskawa matrix is taken to be diagonal. We work with $n_{f}=5$ massless quark flavors and retain the exact top-mass dependence in all virtual and real-virtual amplitudes associated to bottom-induced processes, except for the two-loop virtual corrections, where we neglect top-mass effects. Given the smallness of the bottom-quark density, we estimate the corresponding error to be at the percent level of the computed correction. We use the NNPDF31_nnlo_as_0118_luxqed set of parton distributions [113], which is based on the LUXqed methodology [114] for the determination of the photon density. The renormalization and factorization scales are fixed to $\mu_{R}=\mu_{F}=m_{Z}$, and the corresponding value of $\alpha_{S}$ is set.

We use the following selection cuts on the transverse momenta and rapidities of the muons $p_{T, \mu^{ \pm}}$and $y_{\mu^{ \pm}}$and on the invariant mass $m_{\mu \mu}$ of the dimuon pair

$$
p_{T, \mu^{ \pm}}>25 \mathrm{GeV}, \quad\left|y_{\mu^{ \pm}}\right|<2.5, \quad m_{\mu \mu}>50 \mathrm{GeV} .
$$

We work at the level of bare muons, i.e., no lepton recombination with close-by photons is carried out.

We start the presentation of our results with the fiducial cross section. In Table I we report the contributions $\sigma^{(i, j)}$ to the cross section [see Eq. (2)] in the various partonic channels. The numerical uncertainties are stated in brackets, and for the NNLO corrections $\sigma^{(2,0)}$ and the mixed QCD-EW contributions $\sigma^{(1,1)}$ they include the systematic uncertainties that will be discussed below. The contribution from quark-antiquark annihilation is denoted by $q \bar{q}$. The 
TABLE I. The different perturbative contributions to the fiducial cross section [see Eq. (2)]. The breakdown into the various partonic channels is also shown (see text).

\begin{tabular}{lccccc}
\hline \hline$\sigma[\mathrm{pb}]$ & $\sigma_{\mathrm{LO}}$ & $\sigma^{(1,0)}$ & $\sigma^{(0,1)}$ & $\sigma^{(2,0)}$ & $\sigma^{(1,1)}$ \\
\hline$q \bar{q}$ & $809.56(1)$ & $191.85(1)$ & $-33.76(1)$ & $49.9(7)$ & $-4.8(3)$ \\
$q g$ & & $-158.08(2)$ & & $-74.8(5)$ & $8.6(1)$ \\
$q(g) \gamma$ & & & $-0.839(2)$ & & $0.084(3)$ \\
$q(\bar{q}) q^{\prime}$ & & & & $6.3(1)$ & $0.19(0)$ \\
$g g$ & & & & $18.1(2)$ & \\
$\gamma \gamma$ & $1.42(0)$ & & $-0.0117(4)$ & & \\
tot & $810.98(1)$ & $33.77(2)$ & $-34.61(1)$ & $-0.5(9)$ & $4.0(3)$ \\
\hline \hline
\end{tabular}

contributions from the channels $q g+\bar{q} g$ and $q \gamma+\bar{q} \gamma+g \gamma$ are labeled by $q g$ and $q(g) \gamma$, respectively. The contribution from all the remaining quark-quark channels $q q^{\prime}, \bar{q} \bar{q}^{\prime}$ (including both $q=q^{\prime}$ and $q \neq q^{\prime}$ ), and $q \bar{q}^{\prime}$ (with $\left.q \neq q^{\prime}\right)$ is labeled by $q(\bar{q}) q^{\prime}$. Finally, the contributions from the gluon-gluon and photon-photon channels are denoted by $g g$ and $\gamma \gamma$, respectively. We see that radiative corrections are subject to large cancellations between the various partonic channels. The NLO QCD corrections $\sigma^{(1,0)}$ amount to $+4.2 \%$ with respect to the $\mathrm{LO}$ result, while the NLO EW correction $\sigma^{(0,1)}$ contributes $-4.3 \%$. Also the NNLO QCD corrections are subject to large cancellations, and give an essentially vanishing contribution within the numerical uncertainties. The newly computed QCD-EW corrections amount to $+0.5 \%$ with respect to the $\mathrm{LO}$ result.

In Fig. 1 we present our result for the $\mathcal{O}\left(\alpha_{S} \alpha\right)$ correction as a function of the antimuon $p_{T}$. The left panels depict the region around the $Z$ peak, and the right panels the high- $p_{T}$ region. In the main panels we show the absolute correction $d \sigma^{(1,1)} / d p_{T}$, while the central (bottom) panels display the correction normalized to the LO (NLO QCD) result. Our results for the complete $\mathcal{O}\left(\alpha_{S} \alpha\right)$ correction are compared with those obtained in two approximations. The first approximation consists of computing the finite part of the two-loop virtual amplitude in the pole approximation, suitably reweighted with the exact squared Born amplitude. This approach precisely follows that adopted for the charged-current DY process in Ref. [51] [see Eq. (14) therein for the precise definition]. The pole approximation, which includes factorizable and nonfactorizable [46] contributions, requires the QCD-EW on shell form factor of the $Z$ boson [42]. The second approximation is based on a fully factorized approach for QCD and EW corrections, where we exclude photon-induced processes throughout (see Ref. [47,51] for a detailed description). We see that the result obtained in the pole approximation is in perfect agreement with the exact result. This is due to the small contribution of the two-loop virtual to the computed correction, as observed also in the case of $W$ production [51]. Our result for the $\mathcal{O}\left(\alpha_{S} \alpha\right)$ correction in the region of the peak is reproduced relatively well by the factorized approximation. Beyond the Jacobian peak, this
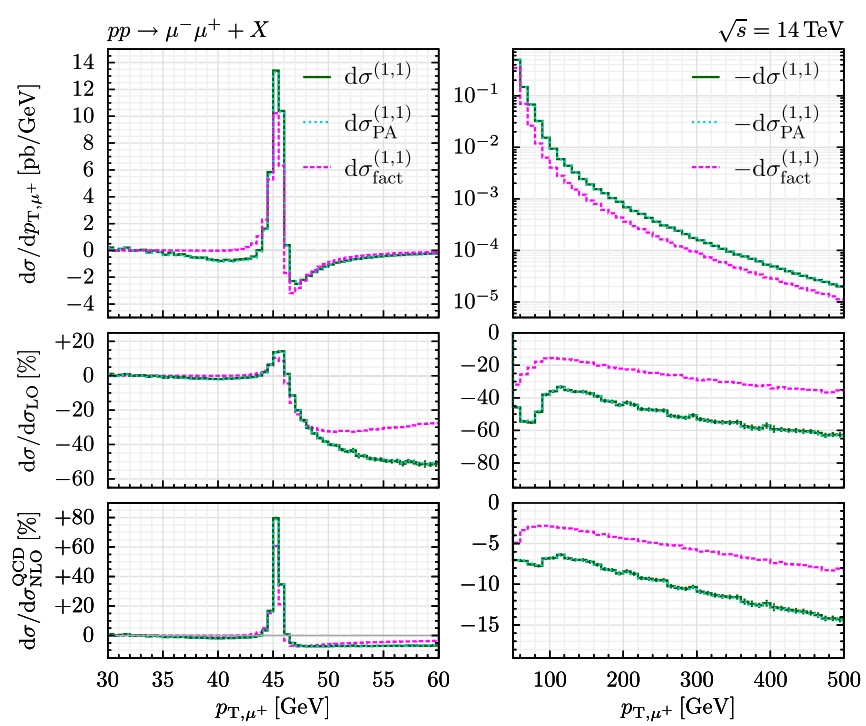

FIG. 1. Complete $\mathcal{O}\left(\alpha_{S} \alpha\right)$ correction to the differential cross section $d \sigma^{(1,1)}$ in the antimuon $p_{T}$ compared with the corresponding result in the pole approximation and the factorized approximation $d \sigma_{\text {fact }}^{(1,1)}$. The top panels show the absolute predictions, while the central (bottom) panels display the $\mathcal{O}\left(\alpha_{S} \alpha\right)$ correction normalized to the LO (NLO QCD) result. For the full result the ratios also display our estimate of the numerical uncertainties, obtained as described in the text.

approximation tends to overshoot the complete result, which is consistent with what was observed in Refs. [47,51]. This is not unexpected, since the high- $p_{T}$ region is dominated by $Z+$ jet topologies, for which the $\mathcal{O}\left(\alpha_{S} \alpha\right)$ effects can be seen as NLO EW corrections. As $p_{T}$ increases, the (negative) impact of the mixed QCD-EW corrections increases, and at $p_{T}=500 \mathrm{GeV}$ it reaches about $-60 \%$ with respect to the LO prediction and $-15 \%$ with respect to the NLO QCD result.

In Fig. 2 we show our result for the $\mathcal{O}\left(\alpha_{S} \alpha\right)$ correction as a function of the dimuon invariant mass $m_{\mu \mu}$. The left panels depict the region around the $Z$ peak, and the right panels the high- $m_{\mu \mu}$ region. When comparing the factorized approximation with the exact result, we notice that it fails to describe the radiative correction below the $Z$ resonance, as already pointed out in Ref. [47]. In contrast, the pole approximation is a very good approximation of the complete correction, with some small differences that can be appreciated right around the peak. In the high $-m_{\mu \mu}$ region the correction is uniformly of the order of $-1.5 \%$ with respect to the NLO QCD result. Here the trend of the negative correction is captured by both approximations, which, however, both undershoot the exact result by about $30 \%$, highlighting the relevance of the exact two-loop contribution for this observable.

The numerical evaluation of the above results for $d \sigma^{(1,1)}$ (and $d \sigma^{(2,0)}$ ) requires the introduction of a technical cut-off $r_{\text {cut }}$ on the dimensionless variable $q_{T} / m_{\mu \mu}$ in the 

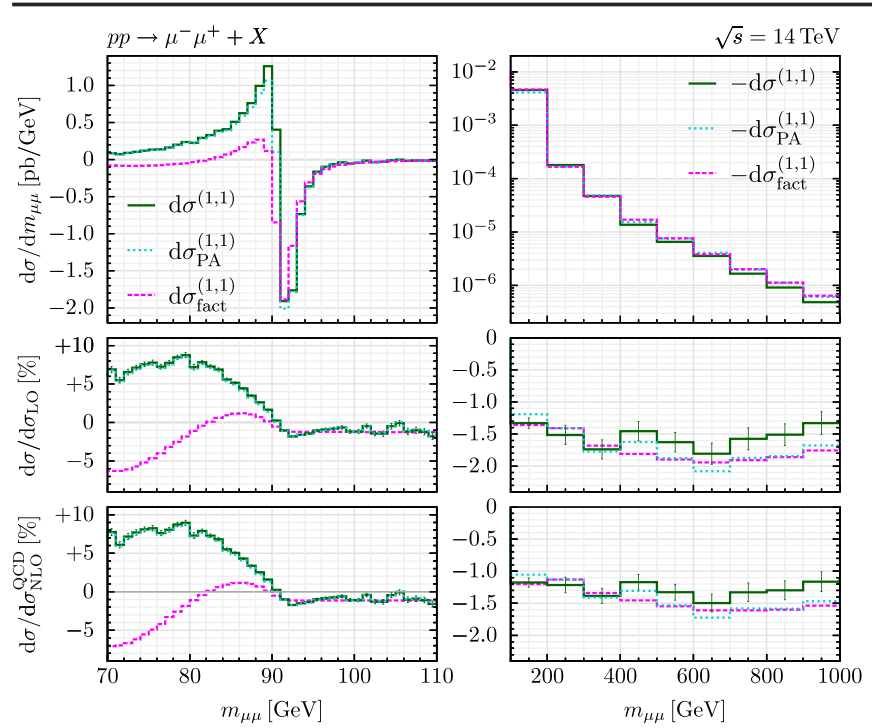

FIG. 2. As Fig. 1, but for the dimuon invariant mass.

square bracket of Eq. (3). We follow the procedure in MATRIX [106] to simultaneously calculate for several values of $r_{\text {cut }}$ and to perform a numerical extrapolation $r_{\text {cut }} \rightarrow 0$, but apply it on a bin-wise level. Quadratic least $\chi^{2}$ fits in the range $\left[0.01 \%, r_{\max }\right]$ with $r_{\max } \in[0.25 \%, 0.5 \%]$ are used to determine best predictions and extrapolation error estimates. In the case of the antimuon $p_{T}$ distribution, the final uncertainties of the computed correction, combining statistical and systematic errors, range from the percent level in the peak region to $\mathcal{O}(3 \%)$ in the tail. In the case of the dimuon invariant mass, the final uncertainties are larger, and range from the few percent level in the peak region to $\mathcal{O}(10 \%)$ at high $m_{\mu \mu}$ values.

Summary.-In this Letter we have presented the first complete computation of the mixed QCD-EW corrections to neutral-current DY lepton pair production at the LHC. All the real and virtual contributions due to initial- and final-state radiation are included exactly, thereby allowing us to investigate the impact of the computed corrections in the entire region of dilepton invariant masses. The evaluation of the two-loop virtual amplitude has been achieved by using semianalytical techniques. To cancel soft and collinear singularities, we have used a formulation of the $q_{T}$ subtraction formalism derived from the NNLO QCD calculation for heavy-quark production through an appropriate abelianization procedure. Our computation is fully differential in the momenta of the charged leptons and the associated QED and QCD radiation. Therefore, it can be used to compute arbitrary infrared-safe observables, and, in particular, we can also deal with dressed leptons, i.e., leptons recombined with close-by photons. More detailed results of our calculation, and a study of perturbative uncertainties, will be presented elsewhere.

We would like to express our gratitude to Jean-Nicolas Lang and Jonas Lindert for their continuous support on
RECOLA and OPENLOOPS, to Simone Devoto for fruitful discussions and several checks of the two-loop amplitudes, and to Chiara Savoini for numerical checks of the pole approximation. This work is supported in part by the Swiss National Science Foundation (SNF) under Contract No. 200020_188464. The work of S. K. is supported by the ERC Starting Grant No. 714788 REINVENT. F. T. acknowledges support from INFN. A. V. and N. R. are supported by the Italian Ministero dell'Università e della Ricerca (Grant No. PRIN2017) and by the European Research Council under the European Unions Horizon 2020 research and innovation program (Grant Agreement No. 740006). R. B. and N.R. acknowledge the COST (European Cooperation in Science and Technology) Action CA16201 PARTICLEFACE for partial support.

*roberto.bonciani@roma1.infn.it

†lbuono@physik.uzh.ch

‡razzini@physik.uzh.ch

\$stefan.kallweit@cern.ch

"narayan.rana@mi.infn.it

"tramonta@na.infn.it

Alessandro.Vicini@mi.infn.it

[1] G. Aad et al. (ATLAS Collaboration), Observation of a new particle in the search for the standard model Higgs boson with the ATLAS detector at the LHC, Phys. Lett. B 716, 1 (2012).

[2] S. Chatrchyan et al. (CMS Collaboration), Observation of a new boson at a mass of $125 \mathrm{GeV}$ with the CMS experiment at the LHC, Phys. Lett. B 716, 30 (2012).

[3] S. Drell and T.-M. Yan, Massive Lepton Pair Production in Hadron-Hadron Collisions at High-Energies, Phys. Rev. Lett. 25, 316 (1970); Erratum, Phys. Rev. Lett. 25, 902 (1970).

[4] G. Arnison et al. (UA1 Collaboration), Experimental observation of isolated large transverse energy electrons with associated missing energy at $\sqrt{s}=540 \mathrm{GeV}$, Phys. Lett. 122B, 103 (1983).

[5] M. Banner et al. (UA2 Collaboration), Observation of single isolated electrons of high transverse momentum in events with missing transverse energy at the CERN anti-p p collider, Phys. Lett. 122B, 476 (1983).

[6] G. Arnison et al. (UA1 Collaboration), Experimental observation of lepton pairs of invariant mass around $95-\mathrm{GeV} / \mathrm{c}^{2}$ at the CERN SPS collider, Phys. Lett. 126B, 398 (1983).

[7] P. Bagnaia et al. (UA2 Collaboration), Evidence for $Z^{0} \rightarrow e^{+} e^{-}$at the CERN $\bar{p} p$ collider, Phys. Lett. 129B, 130 (1983).

[8] Tevatron Electroweak Working Group, CDF, D0 Collaborations, 2012 update of the combination of CDF and D0 results for the mass of the $\mathrm{W}$ boson, arXiv:1204.0042.

[9] M. Aaboud et al. (ATLAS Collaboration), Measurement of the $W$-boson mass in pp collisions at $\sqrt{s}=7 \mathrm{TeV}$ with the ATLAS detector, Eur. Phys. J. C 78, 110 (2018); Erratum, Eur. Phys. J. C 78, 898 (2018). 
[10] T. A. Aaltonen et al. (CDF, D0 Collaborations), Tevatron Run II combination of the effective leptonic electroweak mixing angle, Phys. Rev. D 97, 112007 (2018).

[11] Measurement of the effective leptonic weak mixing angle using electron and muon pairs from $Z$-boson decay in the ATLAS experiment at $\sqrt{s}=8 \mathrm{TeV}$, Report No. ATLASCONF-2018-037, 2018, http://cds.cern.ch/record/ 2630340/files/ATLAS-CONF-2018-037.pdf?version=1.

[12] G. Altarelli, R. Ellis, and G. Martinelli, Large perturbative corrections to the Drell-Yan process in QCD, Nucl. Phys. B157, 461 (1979).

[13] R. Hamberg, W. van Neerven, and T. Matsuura, A complete calculation of the order $\alpha_{s}^{2}$ correction to the Drell-Yan $K$ factor, Nucl. Phys. B359, 343 (1991); Erratum, Nucl. Phys. B644, 403 (2002).

[14] R. V. Harlander and W. B. Kilgore, Next-to-Next-toLeading Order Higgs Production at Hadron Colliders, Phys. Rev. Lett. 88, 201801 (2002).

[15] C. Anastasiou, L. J. Dixon, K. Melnikov, and F. Petriello, Dilepton Rapidity Distribution in the Drell-Yan Process at NNLO in QCD, Phys. Rev. Lett. 91, 182002 (2003).

[16] C. Anastasiou, L. J. Dixon, K. Melnikov, and F. Petriello, High precision QCD at hadron colliders: Electroweak gauge boson rapidity distributions at NNLO, Phys. Rev. D 69, 094008 (2004).

[17] K. Melnikov and F. Petriello, Electroweak gauge boson production at hadron colliders through $\mathcal{O}\left(\alpha_{s}^{2}\right)$, Phys. Rev. D 74, 114017 (2006).

[18] S. Catani, L. Cieri, G. Ferrera, D. de Florian, and M. Grazzini, Vector Boson Production at Hadron Colliders: A Fully Exclusive QCD Calculation at NNLO, Phys. Rev. Lett. 103, 082001 (2009).

[19] S. Catani, G. Ferrera, and M. Grazzini, W boson production at hadron colliders: The lepton charge asymmetry in NNLO QCD, J. High Energy Phys. 05 (2010) 006.

[20] S. Dittmaier and M. Krämer, Electroweak radiative corrections to $\mathrm{W}$ boson production at hadron colliders, Phys. Rev. D 65, 073007 (2002).

[21] U. Baur and D. Wackeroth, Electroweak radiative corrections to $p \bar{p} \rightarrow W^{ \pm} \rightarrow \ell^{ \pm} \nu$ beyond the pole approximation, Phys. Rev. D 70, 073015 (2004).

[22] V. Zykunov, Radiative corrections to the Drell-Yan process at large dilepton invariant masses, Phys. At. Nucl. 69, 1522 (2006).

[23] A. Arbuzov, D. Bardin, S. Bondarenko, P. Christova, L. Kalinovskaya, G. Nanava, and R. Sadykov, One-loop corrections to the Drell-Yan process in SANC. I. The charged current case, Eur. Phys. J. C 46, 407 (2006); Erratum, Eur. Phys. J. C 50, 505 (2007).

[24] C. Carloni Calame, G. Montagna, O. Nicrosini, and A. Vicini, Precision electroweak calculation of the charged current Drell-Yan process, J. High Energy Phys. 12 (2006) 016.

[25] U. Baur, O. Brein, W. Hollik, C. Schappacher, and D. Wackeroth, Electroweak radiative corrections to neutral current Drell-Yan processes at hadron colliders, Phys. Rev. D 65, 033007 (2002).

[26] V. A. Zykunov, Weak radiative corrections to Drell-Yan process for large invariant mass of di-lepton pair, Phys. Rev. D 75, 073019 (2007).
[27] C. Carloni Calame, G. Montagna, O. Nicrosini, and A. Vicini, Precision electroweak calculation of the production of a high transverse-momentum lepton pair at hadron colliders, J. High Energy Phys. 10 (2007) 109.

[28] A. Arbuzov, D. Bardin, S. Bondarenko, P. Christova, L. Kalinovskaya, G. Nanava, and R. Sadykov, One-loop corrections to the Drell-Yan process in SANC. (II). The neutral current case, Eur. Phys. J. C 54, 451 (2008).

[29] S. Dittmaier and M. Huber, Radiative corrections to the neutral-current Drell-Yan process in the standard model and its minimal supersymmetric extension, J. High Energy Phys. 01 (2010) 060.

[30] C. Duhr, F. Dulat, and B. Mistlberger, Drell-Yan Cross Section to Third Order in the Strong Coupling Constant, Phys. Rev. Lett. 125, 172001 (2020).

[31] X. Chen, T. Gehrmann, N. Glover, A. Huss, T.-Z. Yang, and H. X. Zhu, Di-lepton rapidity distribution in Drell-Yan production to third order in QCD, arXiv:2107.09085.

[32] C. Duhr, F. Dulat, and B. Mistlberger, Charged current Drell-Yan production at $\mathrm{N}^{3} \mathrm{LO}$, J. High Energy Phys. 11 (2020) 143.

[33] S. Camarda, L. Cieri, and G. Ferrera, Drell-Yan lepton-pair production: $q_{T}$ resummation at $\mathrm{N}^{3} \mathrm{LL}$ accuracy and fiducial cross sections at $\mathrm{N}^{3}$ LO, arXiv:2103.04974 [Phys. Rev. D (to be published)].

[34] L. Barze, G. Montagna, P. Nason, O. Nicrosini, F. Piccinini, and A. Vicini, Neutral current Drell-Yan with combined QCD and electroweak corrections in the POWHEG BOX, Eur. Phys. J. C 73, 2474 (2013).

[35] S. Kallweit, J. M. Lindert, P. Maierhofer, S. Pozzorini, and M. Schönherr, NLO QCD + EW predictions for $\mathrm{V}+$ jets including off-shell vector-boson decays and multijet merging, J. High Energy Phys. 04 (2016) 021.

[36] J. R. Andersen et al., Les Houches 2015: Physics at TeV colliders standard model working group report, in 9th Les Houches Workshop on Physics at TeV Colliders (2016).

[37] D. de Florian, M. Der, and I. Fabre, QCD $\oplus$ QED NNLO corrections to Drell Yan production, Phys. Rev. D 98, 094008 (2018).

[38] L. Cieri, D. de Florian, M. Der, and J. Mazzitelli, Mixed $\mathrm{QCD} \otimes \mathrm{QED}$ corrections to exclusive Drell Yan production using the $\mathrm{q}_{T}$-subtraction method, J. High Energy Phys. 09 (2020) 155.

[39] M. Delto, M. Jaquier, K. Melnikov, and R. Röntsch, Mixed $\mathrm{QCD} \otimes \mathrm{QED}$ corrections to on-shell $Z$ boson production at the LHC, J. High Energy Phys. 01 (2020) 043.

[40] R. Bonciani, F. Buccioni, R. Mondini, and A. Vicini, Double-real corrections at $\mathcal{O}\left(\alpha \alpha_{s}\right)$ to single gauge boson production, Eur. Phys. J. C 77, 187 (2017).

[41] R. Bonciani, F. Buccioni, N. Rana, I. Triscari, and A. Vicini, NNLO QCD $\times$ EW corrections to $\mathrm{Z}$ production in the $q \bar{q}$ channel, Phys. Rev. D 101, 031301(R) (2020).

[42] R. Bonciani, F. Buccioni, N. Rana, and A. Vicini, NNLO QCD $\times$ EW Corrections to On-Shell Z Production, Phys. Rev. Lett. 125, 232004 (2020).

[43] F. Buccioni, F. Caola, M. Delto, M. Jaquier, K. Melnikov, and R. Röntsch, Mixed QCD-electroweak corrections to on-shell Z production at the LHC, Phys. Lett. B 811, 135969 (2020). 
[44] A. Behring, F. Buccioni, F. Caola, M. Delto, M. Jaquier, K. Melnikov, and R. Röntsch, Mixed QCD-electroweak corrections to $W$-boson production in hadron collisions, Phys. Rev. D 103, 013008 (2021).

[45] A. Denner and S. Dittmaier, Electroweak radiative corrections for collider physics, Phys. Rep. 864, 1 (2020).

[46] S. Dittmaier, A. Huss, and C. Schwinn, Mixed QCDelectroweak $\mathcal{O}\left(\alpha_{s} \alpha\right)$ corrections to Drell-Yan processes in the resonance region: Pole approximation and nonfactorizable corrections, Nucl. Phys. B885, 318 (2014).

[47] S. Dittmaier, A. Huss, and C. Schwinn, Dominant mixed QCD-electroweak $\mathrm{O}\left(\alpha_{s} \alpha\right)$ corrections to Drell-Yan processes in the resonance region, Nucl. Phys. B904, 216 (2016).

[48] C. M. Carloni Calame, M. Chiesa, H. Martinez, G. Montagna, O. Nicrosini, F. Piccinini, and A. Vicini, Precision measurement of the W-boson mass: Theoretical contributions and uncertainties, Phys. Rev. D 96, 093005 (2017).

[49] A. Behring, F. Buccioni, F. Caola, M. Delto, M. Jaquier, K. Melnikov, and R. Röntsch, Estimating the impact of mixed QCD-electroweak corrections on the $W$-mass determination at the LHC, Phys. Rev. D 103, 113002 (2021).

[50] S. Dittmaier, T. Schmidt, and J. Schwarz, Mixed NNLO QCD $\times$ electroweak corrections of $\mathcal{O}\left(N_{f} \alpha_{s} \alpha\right)$ to single$W / Z$ production at the LHC, J. High Energy Phys. 12 (2020) 201.

[51] L. Buonocore, M. Grazzini, S. Kallweit, C. Savoini, and F. Tramontano, Mixed QCD-EW corrections to $p p \rightarrow$ $\ell \nu_{\ell}+X$ at the LHC, Phys. Rev. D 103, 114012 (2021).

[52] R. Bonciani, S. Di Vita, P. Mastrolia, and U. Schubert, Two-loop master integrals for the mixed EW-QCD virtual corrections to Drell-Yan scattering, J. High Energy Phys. 09 (2016) 091.

[53] M. Heller, A. von Manteuffel, and R. M. Schabinger, Multiple polylogarithms with algebraic arguments and the two-loop EW-QCD Drell-Yan master integrals, Phys. Rev. D 102, 016025 (2020).

[54] S. M. Hasan and U. Schubert, Master Integrals for the mixed QCD-QED corrections to the Drell-Yan production of a massive lepton pair, J. High Energy Phys. 11 (2020) 107.

[55] R. Bonciani, A. Ferroglia, T. Gehrmann, D. Maitre, and C. Studerus, Two-loop fermionic corrections to heavy-quark pair production: The quark-antiquark channel, J. High Energy Phys. 07 (2008) 129.

[56] R. Bonciani, A. Ferroglia, T. Gehrmann, and C. Studerus, Two-loop planar corrections to heavy-quark pair production in the quark-antiquark channel, J. High Energy Phys. 08 (2009) 067.

[57] P. Mastrolia, M. Passera, A. Primo, and U. Schubert, Master integrals for the NNLO virtual corrections to $\mu e$ scattering in QED: The planar graphs, J. High Energy Phys. 11 (2017) 198.

[58] M. Heller, A. von Manteuffel, R. M. Schabinger, and H. Spiesberger, Mixed EW-QCD two-loop amplitudes for $q \bar{q} \rightarrow \ell^{+} \ell^{-}$and $\gamma_{5}$ scheme independence of multi-loop corrections, J. High Energy Phys. 05 (2021) 213.

[59] F. Cascioli, P. Maierhöfer, and S. Pozzorini, Scattering Amplitudes with Open Loops, Phys. Rev. Lett. 108, 111601 (2012).
[60] F. Buccioni, S. Pozzorini, and M. Zoller, On-the-fly reduction of open loops, Eur. Phys. J. C 78, 70 (2018).

[61] F. Buccioni, J.-N. Lang, J. M. Lindert, P. Maierhöfer, S. Pozzorini, H. Zhang, and M. F. Zoller, OpenLoops 2, Eur. Phys. J. C 79, 866 (2019).

[62] S. Actis, A. Denner, L. Hofer, J.-N. Lang, A. Scharf, and S. Uccirati, RECOLA: REcursive computation of one-loop amplitudes, Comput. Phys. Commun. 214, 140 (2017).

[63] A. Denner, J.-N. Lang, and S. Uccirati, Recola2: REcursive computation of one-loop amplitudes 2, Comput. Phys. Commun. 224, 346 (2018).

[64] A. Denner, S. Dittmaier, and L. Hofer, Collier: A fortranbased complex one-loop library in extended regularizations, Comput. Phys. Commun. 212, 220 (2017).

[65] P. Nogueira, Automatic Feynman graph generation, J. Comput. Phys. 105, 279 (1993).

[66] J. A. M. Vermaseren, New features of FORM, arXiv:math$\mathrm{ph} / 0010025$.

[67] D. Kreimer, The $\gamma(5)$ problem and anomalies: A clifford algebra approach, Phys. Lett. B 237, 59 (1990).

[68] S. A. Larin, The renormalization of the axial anomaly in dimensional regularization, Phys. Lett. B 303, 113 (1993).

[69] F. V. Tkachov, A theorem on analytical calculability of four loop renormalization group functions, Phys. Lett. 100B, 65 (1981).

[70] K. G. Chetyrkin and F. V. Tkachov, Integration by parts: The algorithm to calculate beta functions in 4 loops, Nucl. Phys. B192, 159 (1981).

[71] T. Gehrmann and E. Remiddi, Differential equations for two loop four point functions, Nucl. Phys. B580, 485 (2000).

[72] P. Maierhöfer, J. Usovitsch, and P. Uwer, Kira-A Feynman integral reduction program, Comput. Phys. Commun. 230, 99 (2018).

[73] R. N. Lee, Presenting LiteRed: A tool for the loop InTEgrals REDuction, arXiv:1212.2685.

[74] C. Studerus, Reduze-Feynman integral reduction in $\mathrm{C}++$, Comput. Phys. Commun. 181, 1293 (2010).

[75] A. von Manteuffel and C. Studerus, Reduze 2-Distributed Feynman integral reduction, arXiv:1201.4330.

[76] A. Goncharov, Polylogarithms in arithmetic and geometry, Proc. Internat. Congress Math. 1,2, 374 (1995).

[77] A. Goncharov, Multiple polylogarithms and mixed Tate motives, arXiv:math/0103059.

[78] E. Remiddi and J. Vermaseren, Harmonic polylogarithms, Int. J. Mod. Phys. A 15, 725 (2000).

[79] K.-T. Chen, Iterated path integrals, Bull. Am. Math. Soc. 83, 831 (1977).

[80] F. Moriello, Generalised power series expansions for the elliptic planar families of Higgs + jet production at two loops, J. High Energy Phys. 01 (2020) 150.

[81] M. Hidding, DiffExp, a Mathematica package for computing Feynman integrals in terms of one-dimensional series expansions, Comput. Phys. Commun. 269, 108125 (2021).

[82] J. Vollinga and S. Weinzierl, Numerical evaluation of multiple polylogarithms, Comput. Phys. Commun. 167, 177 (2005).

[83] A. V. Smirnov, FIESTA4: Optimized Feynman integral calculations with GPU support, Comput. Phys. Commun. 204, 189 (2016). 
[84] S. Borowka, G. Heinrich, S. Jahn, S. P. Jones, M. Kerner, J. Schlenk, and T. Zirke, pySecDec: A toolbox for the numerical evaluation of multi-scale integrals, Comput. Phys. Commun. 222, 313 (2018).

[85] We evaluated the 36 MIs of Ref. [52] with DIFFEXP. Since the first 31 MIs are also available in closed analytical form in terms of generalized polylogarithms, we checked them requesting 30 digits accuracy in GINAC, finding perfect agreement. This independent validation supports our evaluation in the physical region for the last 5 MIs. We also performed additional checks with FIESTA and py SECDEC, with percent precision in the nonphysical region for all the MIs, and with 5-10\% precision in the physical region for the first 31 MIs.

[86] We have explicitly checked the cancellation of the collinear divergences arising in the box diagrams with a photon exchanged between the quark and lepton lines [87].

[87] J. Frenkel and J.C. Taylor, Exponentiation of leading infrared divergences in massless Yang-Mills theories, Nucl. Phys. B116, 185 (1976).

[88] A. Denner, G. Weiglein, and S. Dittmaier, Application of the background field method to the electroweak standard model, Nucl. Phys. B440, 95 (1995).

[89] A. Sirlin, Radiative corrections in the SU(2)-L x U(1) theory: A simple renormalization framework, Phys. Rev. D 22, 971 (1980).

[90] G. Degrassi and A. Vicini, Two loop renormalization of the electric charge in the standard model, Phys. Rev. D 69, 073007 (2004).

[91] B. A. Kniehl, Two loop corrections to the vacuum polarizations in perturbative QCD, Nucl. Phys. B347, 86 (1990).

[92] A. Djouadi and P. Gambino, Electroweak gauge bosons selfenergies: Complete QCD corrections, Phys. Rev. D 49, 3499 (1994); Erratum, Phys. Rev. D 53, 4111 (1996).

[93] S. Catani and M. Grazzini, An NNLO Subtraction Formalism in Hadron Collisions and its Application to Higgs Boson Production at the LHC, Phys. Rev. Lett. 98, 222002 (2007).

[94] S. Catani, S. Devoto, M. Grazzini, S. Kallweit, J. Mazzitelli, and H. Sargsyan, Top-quark pair hadroproduction at next-to-next-to-leading order in QCD, Phys. Rev. D 99, 051501(R) (2019).

[95] S. Catani, S. Devoto, M. Grazzini, S. Kallweit, and J. Mazzitelli, Top-quark pair production at the LHC: Fully differential QCD predictions at NNLO, J. High Energy Phys. 07 (2019) 100.

[96] S. Catani, S. Devoto, M. Grazzini, S. Kallweit, and J. Mazzitelli, Bottom-quark production at hadron colliders: Fully differential predictions in NNLO QCD, J. High Energy Phys. 03 (2021) 029.

[97] L. Buonocore, M. Grazzini, and F. Tramontano, The $q_{T}$ subtraction method: Electroweak corrections and power suppressed contributions, Eur. Phys. J. C 80, 254 (2020).
[98] S. Catani and M. H. Seymour, The dipole formalism for the calculation of QCD jet cross-sections at next-to-leading order, Phys. Lett. B 378, 287 (1996).

[99] S. Catani and M. H. Seymour, A general algorithm for calculating jet cross-sections in NLO QCD, Nucl. Phys. B485, 291 (1997); Erratum, Nucl. Phys. B510, 503 (1998).

[100] S. Catani, S. Dittmaier, M. H. Seymour, and Z. Trocsanyi, The dipole formalism for next-to-leading order QCD calculations with massive partons, Nucl. Phys. B627, 189 (2002).

[101] S. Kallweit, J. M. Lindert, S. Pozzorini, and M. Schönherr, NLO QCD + EW predictions for $2 \ell 2 \nu$ diboson signatures at the LHC, J. High Energy Phys. 11 (2017) 120.

[102] S. Dittmaier, A general approach to photon radiation off fermions, Nucl. Phys. B565, 69 (2000).

[103] S. Dittmaier, A. Kabelschacht, and T. Kasprzik, Polarized QED splittings of massive fermions and dipole subtraction for non-collinear-safe observables, Nucl. Phys. B800, 146 (2008).

[104] T. Gehrmann and N. Greiner, Photon radiation with MadDipole, J. High Energy Phys. 12 (2010) 050.

[105] M. Schönherr, An automated subtraction of NLO EW infrared divergences, Eur. Phys. J. C 78, 119 (2018).

[106] M. Grazzini, S. Kallweit, and M. Wiesemann, Fully differential NNLO computations with MATRIX, Eur. Phys. J. C 78, 537 (2018).

[107] MUNICH, which is the abbreviation of "MUlti-chaNnel Integrator at Swiss $(\mathrm{CH})$ precision", is an automated parton-level NLO generator by S. Kallweit.

[108] J. Ablinger, A computer algebra toolbox for harmonic sums related to particle physics, Master's thesis, Linz U., 2009.

[109] J. Ablinger, The package HarmonicSums: Computer algebra and analytic aspects of nested sums, Proc. Sci., LL2014 (2014) 019 [arXiv:1407.6180].

[110] C. Duhr and F. Dulat, PolyLogTools-Polylogs for the masses, J. High Energy Phys. 08 (2019) 135.

[111] A. Denner, S. Dittmaier, M. Roth, and L. H. Wieders, Electroweak corrections to charged-current $\mathrm{e}+\mathrm{e}-\longrightarrow \rightarrow 4$ fermion processes: Technical details and further results, Nucl. Phys. B724, 247 (2005); B854, 504 (2012).

[112] For a technical limitation of the semi-analytical approach, the evaluation of the box-type Feynman diagrams with two internal massive lines has been carried out with real masses of the gauge bosons in the Feynman integrals.

[113] V. Bertone, S. Carrazza, N. P. Hartland, and J. Rojo (NNPDF Collaboration), Illuminating the photon content of the proton within a global PDF analysis, SciPost Phys. 5, 008 (2018).

[114] A. Manohar, P. Nason, G. P. Salam, and G. Zanderighi, How Bright is the Proton? A Precise Determination of the Photon Parton Distribution Function, Phys. Rev. Lett. 117, 242002 (2016). 